\title{
Polarization-shaped laser pulses for improved fluorescence anisotropy contrast
}

Georg Achazi ${ }^{1}$, Nils Hermes $^{1}$, Alexander Patas ${ }^{1}$, Daniel Tolksdorf ${ }^{1}$, and Albrecht Lindinger ${ }^{1}$

Institut für Experimentalphysik, Freie Universität Berlin, Arnimallee 14, D-14195 Berlin, Germany

Received: date / Revised version: date

Abstract We present a method to change fluorescence contrast between two dyes with high fluorescence anisotropy in the same solution under different polarization directions by using polarization-shaped pulses. First a theoretical relationship between contrast, anisotropy and polarization is derivated for this case. Then the wavelength dependent polarization anisotropy and the change of contrast by phase-shaping for a solution of Rhodamine B and Stilbene 3 in glycerol is shown. Finally, the application of the polarizationshaped pulses is demonstrated.

PACS. 42.25.Ja Polarization - 82.50.Pt Multiphoton processes - 82.53.Kp Coherent spectroscopy of atoms and molecules - 87.64.kv Fluorescence

\section{Introduction}

Fluorescence spectroscopy is an important tool for the indirect observation of atomic and molecular processes. Of particular relevance is the fluorescence excitation by multiphoton processes, which allows to exploit nonlinear and interference effects [1]. For example this enables threedimensional imaging by two-photon microscopy for cancer diagnosis [2]. Besides the spectral information the polarization anisotropy is of large interest. It can provide information about properties of a sample like molecular orientation, aggregation, rotational diffusion, and energy migration among chemically identical molecules (homoFRET), which are not accessible by the other observables $[3,4]$.

In microscopy and spectroscopy it is often impossible to avoid fluorophores with partially overlapping absorption spectra. In this case it is feasible to enhance the contrast by selective excitation with phase-shaped laser pulses. It has been demonstrated that this method can give higher contrasts than the use of spectral filters [5]. 
Phase-shaped laser pulses are mainly used in the field of coherent control. In the last years not only the shaping of the spectral phase and amplitude of the pulses but also the full control of their polarization was realized $[6,7]$. It was demonstrated that it is possible to transmit these pulses via optical fibers and use them in a coherent control experiment afterwards [8].

In this paper we report the selective excitation of two fluorescent dyes in solution by a polarization-shaped laser pulse. The pulse consists of two orthogonal linearly polarized subpulses, where the phase of the horizontally polarized subpulse is optimized to excite one dye while the phase of the vertically polarized subpulse is optimized for the other dye. Due to fluorescence anisotropy it is possible to change the measured contrast between both fluorescence signals by using a polarizer before the detector. This is an easily applicable technique which could be used to enhance imaging in medicine and biology.

\section{Theory}

In solution the fluorophore molecules are randomly oriented. If the solution is irradiated with linearly polarized light, most of the excited molecules have transition dipole moments oriented within a range of angles close to the polarization of the light. Because the polarization direction of the fluorescence photons is in most cases again parallel to the transition dipole moment, the fluorescence will then be polarized as well, if the molecules do not lose their orientation by rotation. The usual measure for the polarization of the emitted light is the anisotropy

$$
a=\frac{I_{\|}-I_{\perp}}{I_{\|}+2 I_{\perp}}
$$

with the intensities $I_{\|}$and $I_{\perp}$ being the two components of the fluorescence signal, which are polarized parallel and perpendicular to the exciting light. Which in the following is defined as the horizontal orientation of our lab space.

If the solution of fluorophore molecules is irradiated with polarization shaped pulses, $I_{\|}$and $I_{\perp}$ itself are composed of two components. One from the fluorophores excited by the horizontally polarzied part of the laser pulse $\left(I_{H}\right)$ and one from the fluorophores excited by the vertically polarzied part of the pulse $\left(I_{V}\right)$

$$
\begin{gathered}
I_{\|}=I_{H}+\beta I_{V} \\
I_{\|}=I_{V}+\beta I_{H} .
\end{gathered}
$$

The factor $\beta$ is connected with the anisotropy $a$ by

$$
\beta=\frac{1-a}{2+a}
$$

In a solution containing two fluorophores, the contrast between the fluorescence signals $\left(I_{1}, I_{2}\right)$ of two dyes is defined by

$$
k\left(I_{1}, I_{2}\right)=\frac{I_{1}-I_{2}}{I_{1}+I_{2}}
$$

If the fluorescence signal is detected behind a polarizer, the measured contrast is dependent on the orientation of the polarizer and the anisotropy of the fluorophores. In general the contrasts of the fluorescence light in parallel $\left(k_{\|}\right)$and perpendicular to $\left(k_{\perp}\right)$ polarization orientation can be written as

$$
k_{\|}\left(I_{1}, I_{2}\right)=\frac{I_{1 H}+\beta_{1} I_{1 V}-I_{2 H}-\beta_{2} I_{2 V}}{I_{1 H}+\beta_{1} I_{1 V}+I_{2 H}+\beta_{2} I_{2 V}}
$$




$$
k_{\perp}\left(I_{1}, I_{2}\right)=\frac{I_{1 V}+\beta_{1} I_{1 H}-I_{2 V}-\beta_{2} I_{2 H}}{I_{1 V}+\beta_{1} I_{1 H}+I_{2 V}+\beta_{2} I_{2 H}}
$$

where the subscripts 1 and 2 denote from which of the two fluorophore the light is emitted. The goal of the the experiments presented in this paper is to achieve a large difference

$$
\Delta k\left(I_{1}, I_{2}\right)=\left|k_{\|}\left(I_{1}, I_{2}\right)-k_{\perp}\left(I_{1}, I_{2}\right)\right|
$$

between the contrasts measured for both polarizations. The feasibility of this goal depends on the fluorophores and the applied laser pulses. For a single linear polarized excitation pulse polarized parallel to the lab frame, which means $I_{1 V}=I_{2 V}=0$, Eq. 8 becomes

$$
\Delta k=\left|\frac{2\left(1-k_{\|}^{2}\right)\left(\beta_{2}-\beta_{1}\right)}{\left(\beta_{2}+\beta_{1}\right)+k_{\|}\left(\beta_{2}-\beta_{1}\right)}\right| .
$$

In this case one gets a high $\Delta k$ for $k_{\|} \rightarrow 0$ and if there is a large difference between $\beta_{1}$ and $\beta_{2}$, which means a mixture of fluorophores with high and low anisotropy. In this case the change of the contrast between both polarizations $\Delta k$ can get even larger than the change of the contrasts, which can be achieved by phase-shaping. However, $\Delta k$ vanishes for fluorophores with identical anisotropy.

For polarization shaped pulses one can assume for simplicity $I_{1 H}=I_{2 V}=I_{a}$ and $I_{1 V}=I_{2 H}=I_{b}$. This can be realized in the lab by choosing the pulse shapes and concentration ratios appropriately. In this case Eq. 8 becomes $\Delta k=\left|\frac{2\left(I_{a}^{2}-I_{b}^{2}\right)\left(1-\beta_{1} \beta_{2}\right)}{\left(I_{a}\left(1+\beta_{2}\right)+I_{b}\left(1+\beta_{1}\right)\right)\left(I_{a}\left(1+\beta_{1}\right)+I_{b}\left(1+\beta_{2}\right)\right)}\right|$

Hence, $\Delta k$ does not vanish for $\beta_{1}=\beta_{2}$. But to maximize $\Delta k$ different pulse shapes are required to change the con- trast in both directions, since $\Delta k$ vanishes in this case if $I_{a}=I_{b}$.

\section{Experimental Setup}

The experimental setup is shown in Fig. 1. The source of the laser pulses is a regenerative amplifier (RegA 9050, Coherent) with a repetition rate of $284 \mathrm{kHz}$. The FWHM was set to $24.5 \mathrm{~nm}$ at a central wavelength of $805 \mathrm{~nm}$. The pulses were shaped by a commercial spatial light modulator (SLM-640, Cambridge Research Instruments) with two liquid crystal arrays with optical axes at $\pm 45^{\circ}$ in a $4 f$-setup, consisting of two diffraction gratings of 1200 lines/mm and two cylindrical lenses of $250 \mathrm{~mm}$ focal length. The two arrays of the shaper can be used to generate two perpendicular linear polarized independently shaped pulses by delaying the pulse components polarized parallel to the axes of the arrays with respect to each other. Two half-wave plates (HWP) are added to the setup. One is issued to compensate for different reflectivities in horizontal and vertical polarization. The other HWP is placed directly after the SLM-640 to rotate the polarization of the pulses from $+45^{\circ}$ and $-45^{\circ}$ to $0^{\circ}$ and $90^{\circ}$. For the measurements with only one pulse a polarizer is placed behind the setup to cut out one polarization component. This method of polarization-shaping and its capabilities is described in more detail in [9].

The shaped pulses with pulse energies up to $400 \mathrm{~nJ}$ are focused with a $\mathrm{f}=50 \mathrm{~cm}$-lens into a cuvette filled with the dye-solution. The fluoresence-signal of the dyes is collected using two lenses which focus the light on a 


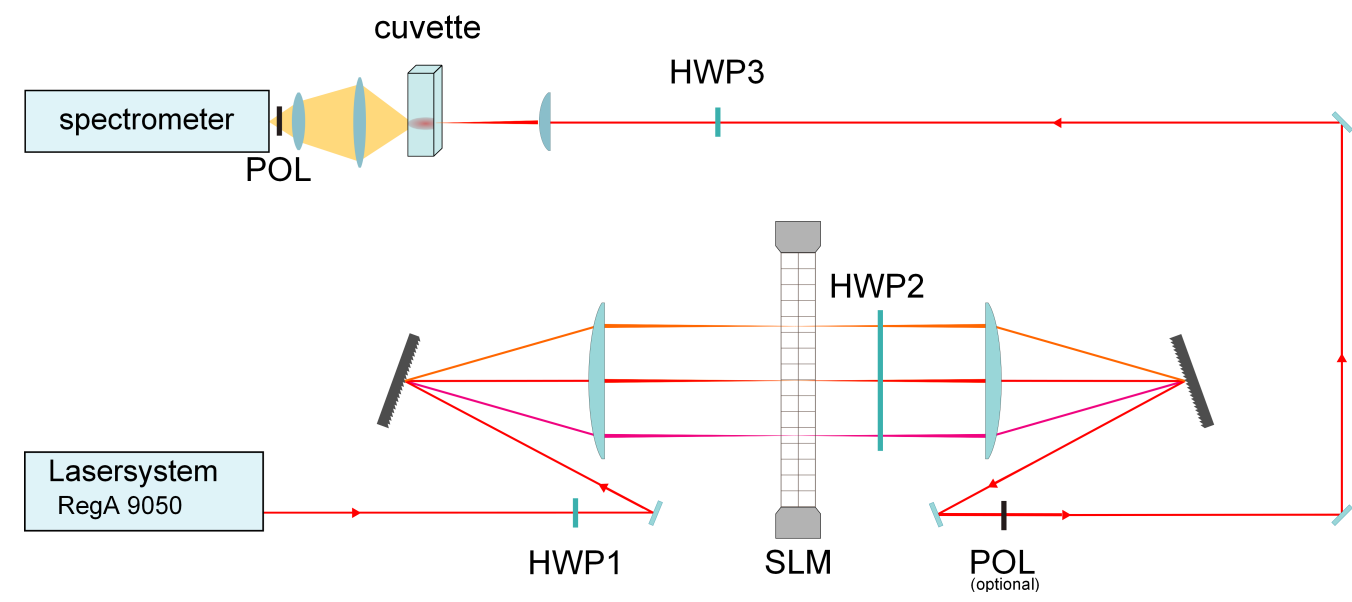

Figure 1. Scheme of the experimental setup.

spectrometer behind a polarizer. Rotating the polarizer slightly changes the beam path and would require to readjust the coupling to the spectrometer after each rotation. Therefore the polarizer is always set at horizontal polarization. To measure the anisotropy a third HWP is used, which has less influence on the beam path when rotating the beam polarization.

The shape of the laser pulses is detected by recording cross-correlation traces for 10 different positions of the third HWP [10]. The same algorithm, which calculates the state of polarization from the cross-correlation traces for each time step, is used to determine the anisotropy for each wavelength from the recorded spectra.

The concentration of dyes in the solution was $4 \mathrm{mM}$ for Stilbene 3 and $0.04 \mathrm{mM}$ for Rhodamine B. The concentrations were chosen to get a similar signal strength for both dyes.

\section{Properties of Stilbene 3 and Rhodamine B}

The experiments are performed on a mixture of the two well known dyes Stilbene 3 and Rhodamine B dissolved in glycerol. These dyes were chosen for several reasons. First of all, both show two-photon absorption around 805 nm but the absorption spectra in Fig. 2 display that the maximum is blue shifted for Stilbene 3 and red shifted for

Rhodamine B. These shifted absorption maxima allow to influence the contrast between the emission of the dyes by phase-shaping. Secondly, the emission spectra of both dyes have very little overlap, therefore it is possible to distinguish between them with a spectrometer. And third, both show high polarization anisotropy in glycerol (Fig. 3b). But while the emission of Rhodamine B has more or less the same polarization for all wavelengths, Stilbene 3 has two overlapping emission bands with perpendicular polarization. This leads to the jump of the orientation curve in Fig. 3c around $460 \mathrm{~nm}$. For the Rhodamine B fluorescence and the longer wavelengths of the Stilbene 3 fluorescence the longer main axis of the polarization ellipse (which de- 


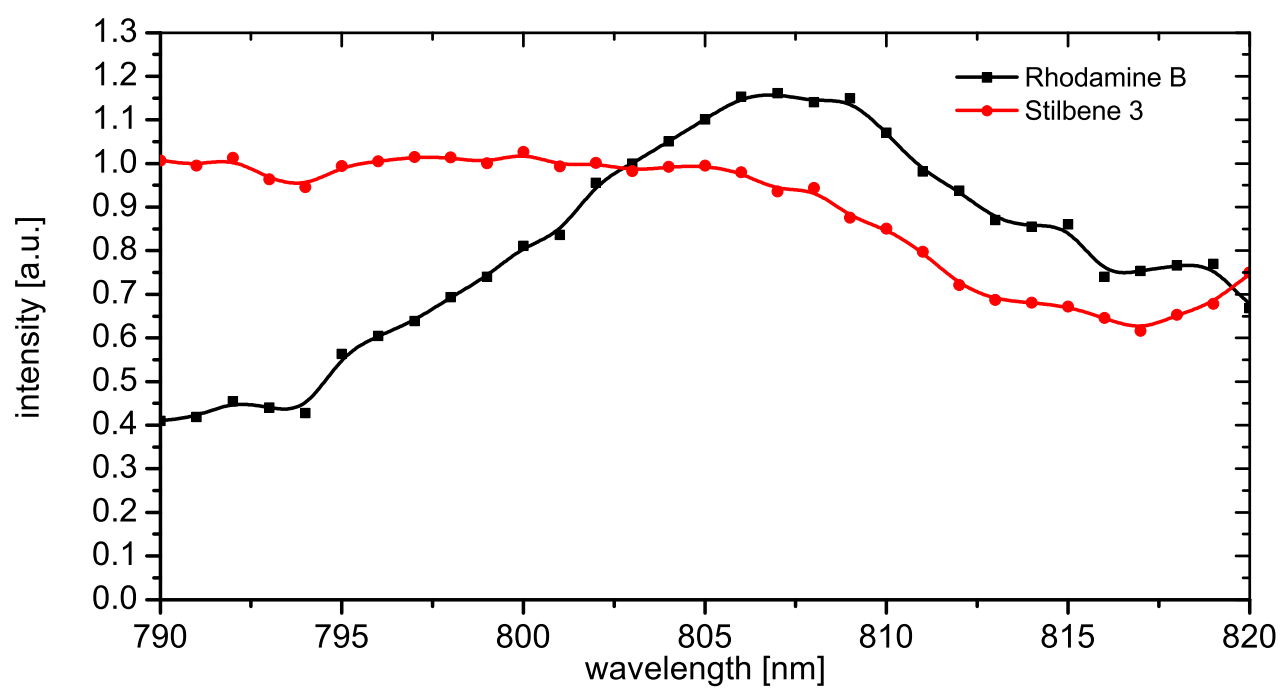

Figure 2. Two-photon absorption spectra of Rhodamine B (black squares) and Stilbene 3 (red circles). The spectra are recorded by using amplitude-shaping with removed HWP1 and HWP2, to generate gaussian spectra with a FWHM of 2.5 nm out of the laser spectrum, detecting the fluorescence in dependence of the central excitation wavelength and dividing the fluorescence by the signal of a two-photon diode for normalization.

fines the orientation) is parallel to the polarization of the exciting laser pulses. Yet, for the shorter wavelengths of the Stilbene 3 spectra this main axis is polarized perpendicular to the exciting light. Because the two emission bands overlap, the measured anisotropy of Stilbene 3 is dependent on the wavelength. At the flanks of the Stilbene 3 spectra the measured anisotropy goes up to 0.6 , but near the center around $460 \mathrm{~nm}$ the anisotropy vanishes. This makes Stilbene 3 a very good test substance because it is possible to "choose" its anisotropy by selecting an appropriate part of the spectra. Especially it is possible to match its anisotropy to that of Rhodamine B by selecting the interval from $520 \mathrm{~nm}$ to $540 \mathrm{~nm}$, which is apparent since both data are close as seen in Fig. 3c. This is the case relevant for the polarization-shaping method.
Further information about the properties of Rhodamine $\mathrm{B}$ and Stilbene 3 can be found in $[11,12,13]$.

\section{Results}

In order to find the pulse shapes leading to a high contrast between both dyes, phases of the form

$$
\varphi(\lambda)=A\left(\frac{c}{\lambda}-\frac{c}{\lambda_{0}}\right)^{3}
$$

are written on the modulator, for different wavelengths $\lambda_{0}$. For each $\lambda_{0}$ the contrast $k$ was calculated by

$$
k=\frac{I_{S t i 3}-I_{R h o B}}{I_{S t i 3}+I_{R h o B}}
$$

Here, $I_{R h o B}$ and $I_{S t i 3}$ are the integrated intensities of the Rhodamine B and Stilbene 3 spectra. This was done separately for both arrays of the modulator. A typical curve for such a measurement is given in Fig. 4. As expected the 

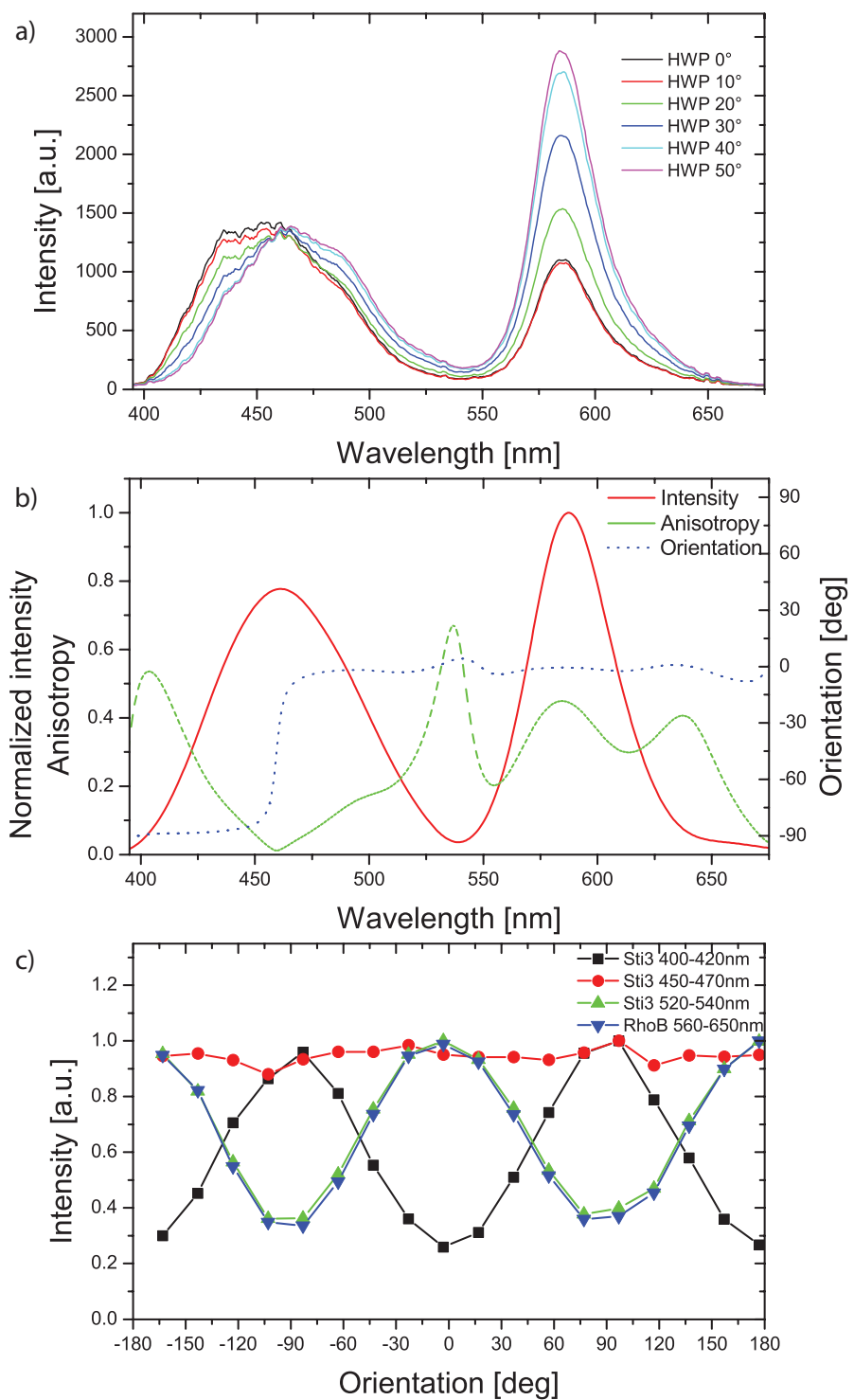

Figure 3. (a) Sample emission spectra of Stilbene 3 and Rhodamine B dissolved in glycerol recorded for different positions of the half-wave plate. (b) Representation of the emission spectra fitted from the spectra shown in (a). The graph gives the intensity integrated over all orientations (solid curve), anisotropy (dashed curve), and orientation of the polarization ellipse (dotted curve) for each wavelength. (c) Normalized intensities in dependence of the polarization for the left (400 - $420 \mathrm{~nm}$, black squares), central (450 - $470 \mathrm{~nm}$, red circles) and right (520 - $540 \mathrm{~nm}$, green triangles) part of the Stilbene 3 spectra and the complete Rhodamine B spectra (560 - $650 \mathrm{~nm}$, blue triangles).

Stilbene 3 fluorescence is higher for shorter wavelengths lectivity, found in these measurements was on the order $\lambda_{0}$ whereas the Rhodamine B fluorescence increases for of 0.3 . It has to be mentioned that the measured conlonger $\lambda_{0}$, due to the shape of the absorption curves. The trast underlies larger deviations for high and low wavemaximal contrast difference, a measure for improved se- lengths due to the vanishing spectral intensity of the ex- 


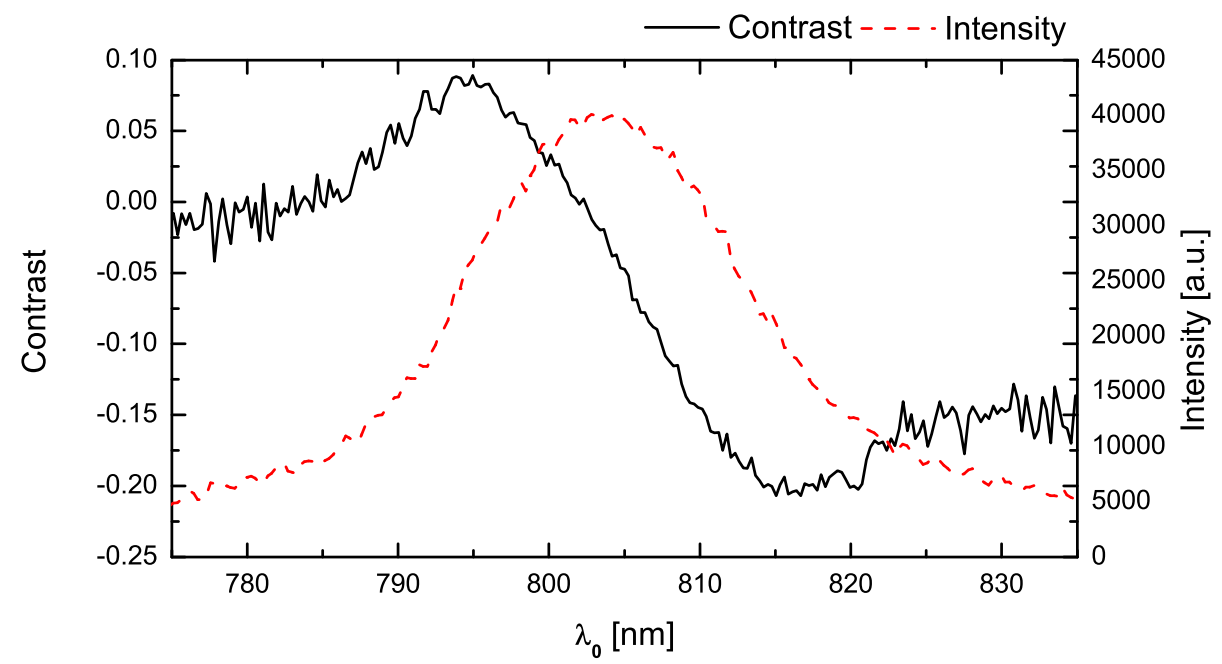

Figure 4. Phase position scan for $A=5 \times 10^{5} \mathrm{fs}^{3}$. The graph gives the contrast between the Rhodamine B and Stilbene 3 intensities (solid curve) and the sum of both intensities (dashed curve).

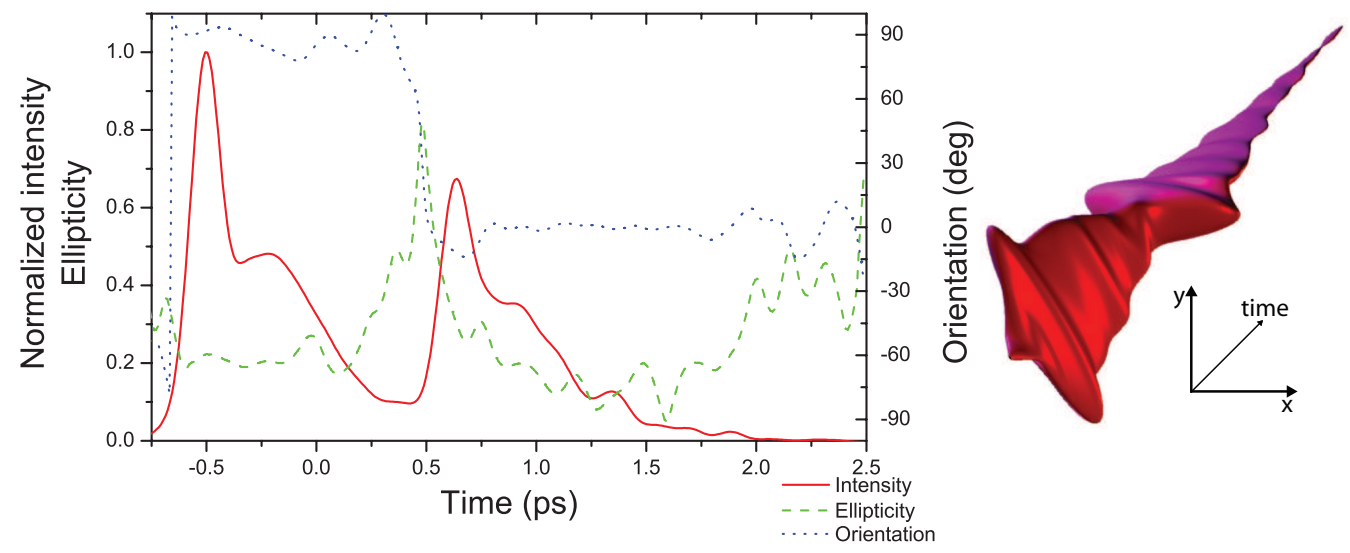

Figure 5. Pulse shape to excite Stilbene 3 in horizontal and Rhodamine B in vertical polarization direction. The graph on the left gives the time evolution of the intensity (solid curve), ellipticity (dashed curve), and orientation (dotted curve) of the pulse. On the right, a three-dimensional representation of the pulse is shown.

citing laser spectrum. This can be indirectly seen from the total fluorescence intensity shown as dashed line in Fig 4. A slightly higher contrast difference can be found by optimizing complex phase functions with a genetic algorithm, but this results in a much weaker fluorescence signal than the simpler phase functions. The parameters used for polarization-shaping are $A=5 \times 10^{5} \mathrm{fs}^{3}, \lambda_{0}=792$ $\mathrm{nm}$ for a horizontally polarized subpulse to efficiently ex- cite Stilbene 3 and $\lambda_{0}=816 \mathrm{~nm}$ for a vertically polarized subpulse to excite Rhodamine B. These parameters were chosen to get a high contrast and to achieve similar intensities in both polarization directions. The temporal shape of the pulse measured by cross-correlation traces is shown in Fig. 5. For this pulse the emission spectra are recorded under different polarization angles. For each direction the signal was integrated over the right flank of the Stilbene 


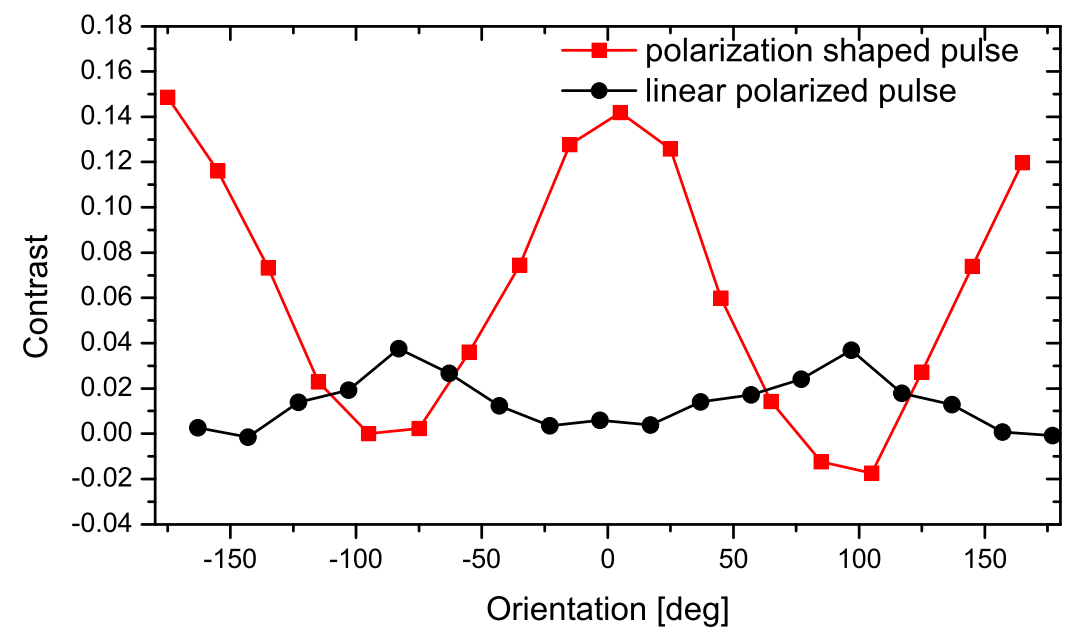

Figure 6. Contrast between the 520 - $540 \mathrm{~nm}$ part of the Stilbene 3 spectrum and the Rhodamine B spectrum for the polarization shaped pulse from Fig. 5 (red squares) and the linear polarized pulse from Fig. 3 (black circles).

3 spectrum (520 - $540 \mathrm{~nm})$ and the whole Rhodamine B $\mathbf{6}$ Conclusion

spectrum (560 - $650 \mathrm{~nm})$. Because of the different sizes

of the integration areas the signals differ by one order of magnitude. Therefore it was necessary to normalize them before calculating the contrast. Each angular scan was divided by the maximum value found by integrating over the corresponding spectral region. The contrasts are shown in Fig. 6 in comparison to the contrasts of a linear polarized pulse measured in the same way. Since the concentrations of the analyzed substances are somehow arbitrary and each detector differs in its sensitivity it has to be said that the result shown in Fig. 6 is of only qualitative significance. While the contrast of the linear pulse stays more or less constant for all angles the contrast of the polarizationshaped pulse varies from -0.02 to 0.15 . This exemplifies the improvement of using polarization shaped laser pulses for two-photon fluorescence.
In this paper improved signal separation of the fluorescence of two dyes with similarly high fluorescence anisotropy was demonstrated. Therefore the anisotropy of the dyes is measured and the change of the contrast between their fluorescence was demonstrated, by the use of simple phase functions. This method could be useful for imaging techniques in biology and medicine. It is not limited to applications involving fluorophores in solutions. It could also be used for imaging of solid samples and in combination with other nonlinear microscopy techniques like secondharmonic imaging microscopy or the probing of nano crystals $[14,15]$.

\section{Acknowledgments}

We thank Prof. Ludger Wöste for his support. 


\section{References}

1. Vadim V. Lozovoy, Igor Pastirk, Katherine A. Walowicz, and Marcos Dantus, The Journal of Chemical Physics 118, (2003) 3187-3196

2. Seth Perry, Ryan Burke, and Edward Brown, Annals of Biomedical Engineering 40, (2012) 277-291

3. Claudiu C. Gradinaru, Denys O. Marushchak, Masood Samim, and Ulrich J. Krull, Analyst 135, (2010) 452-459

4. Andrew H.A. Clayton, Quentin S. Hanley, Donna J. ArndtJovin, Vinod Subramaniam, and Thomas M. Jovin, Biophysical Journal 83, (2002) 1631 - 1649

5. Jennifer P. Ogilvie, Delphine Débarre, Xavier Solinas, JeanLouis Martin, Emmanuel Beaurepaire, and Manuel Joffre, Opt. Express 14, (2006) 759-766

6. M. Ninck, A. Galler, T. Feurer, and T. Brixner, Opt. Lett. 32, (2007) 3379-3381

7. Fabian Weise and Albrecht Lindinger, Opt. Lett. 34, (2009) $1258-1260$

8. Fabian Weise, Georg Achazi, and Albrecht Lindinger, Phys. Chem. Chem. Phys. 13, (2011) 8621-8626

9. Monika Pawłowska, Alexander Patas, Georg Achazi, Nona Rahmat, Fabian Weise, and Albrecht Lindinger, J. Opt. Soc. Am. B 29, (2012) 833-840

10. Mateusz Plewicki, Fabian Weise, Stefan M. Weber, and Albrecht Lindinger, Appl. Opt. 45, (2006) 8354-8359

11. Antonie J. W. G. Visser, Kees Vos, Arie Van Hoek, and Jillert S. Santema, The Journal of Physical Chemistry 92, (1988) 759-765

12. N. Sanz, A. Ibanez, Y. Morel, and P. L. Baldeck, Applied Physics Letters 78, (2001) 2569-2571
13. Mathieu G. Silly, Laurent Porrs, Olivier Mongin, PierreAlain Chollet, and Mireille Blanchard-Desce, Chemical Physics Letters 379, (2003) $74-80$

14. Paul J Campagnola and Leslie M Loew, Nature Biotechnology 21, (2003) $1356-1360$

15. L. Bonacina, Y. Mugnier, F. Courvoisier, R. Le Dantec, J. Extermann, Y. Lambert, V. Boutou, C. Galez, and J.-P. Wolf, Applied Physics B: Lasers and Optics 87, (2007) 399403 